\title{
Mm/Submm images of Herbig-Haro energy sources and candidate protostars
}

\author{
R. Chini ${ }^{1}$, D. Ward-Thompson ${ }^{2}$, J. M. Kirk ${ }^{2}$, M. Nielbock ${ }^{1}$, B. Reipurth ${ }^{3}$, and A. Sievers ${ }^{4}$ \\ 1 Astronomisches Institut der Ruhr-Universität Bochum, Universitätsstr. 150/NA 7, 44780 Bochum, Germany \\ 2 Department of Physics and Astronomy, Cardiff University, PO Box 913, Cardiff, UK \\ 3 CASA, University of Colorado, CB 389, Boulder, CO 80309, USA \\ 4 IRAM, Avda. Divina Pastora 7, Nucleo Central, 18012 Granada, Spain
}

Received 19 September 2000 / Accepted 11 January 2001

\begin{abstract}
We present 450, $850 \mu \mathrm{m}$ and $1300 \mu \mathrm{m}$ images of regions with embedded candidate protostars. Some of them are associated with HH objects (HH 7-11, HH 1-2, HH 147, HH 111, HH 108) and have been previously identified in the course of a $1300 \mu \mathrm{m}$ survey. Other regions were taken from the IRAS PSC $(04239+2436,04368+2557$, $20050+2720,20386+6751,22134+5834,23011+6126)$. The new $\mathrm{mm} / \mathrm{submm}$ images show the detailed structure of the regions some of which contain new compact sources as well as extended emission features. The inferred $\mathrm{mm} / \mathrm{submm}$ fluxes are combined with IRAS data in order to derive the temperature of the associated dust, its mass and the re-radiated luminosity. Taking the ratio of FIR-to-submm luminosity as an indicator for the evolutionary stage, we find that 15 out of 17 sources have $L_{\mathrm{FIR}} / L_{\mathrm{smm}}<200$, indicating that most objects are probably genuine protostars. For the first time, we detect dust emission associated with $\mathrm{HH}$ objects themselves, $\mathrm{H}_{2}$ and CO flows which we interpret as density enhancements swept up by the ejected material.
\end{abstract}

Key words. interstellar medium: dust - stars: circumstellar matter - formation

\section{Introduction}

Regions of star formation contain many tell-tale signs which indicate recent or ongoing formation of new stars. Among the most ubiquitous of such indicators are HerbigHaro (HH) objects. These are shocks which trace the bipolar outflows that all young stellar objects (YSOs) appear to produce shortly after their birth. We have been carrying out a survey of the environments of $\mathrm{HH}$ objects for a number of years at $\mathrm{mm}$ and submm wavelengths (e.g. Chini et al. 1993, 1997; Reipurth et al. 1993, 1996). In the course of this work, we have studied the detailed circumstellar dust structures around the YSOs responsible for driving the outflows associated with the $\mathrm{HH}$ objects (e.g. Chini et al. 1997). In addition, we have also discovered new sources in the vicinity of these YSOs that were not detected by any other means. The best known of these sources is HH 24 MMS (Chini et al. 1993; Ward-Thompson et al. 1995), which appears to be a protostellar object.

Our mm catalogue of new and previously known sources (Chini et al. 1997) can be augmented by $I R A S$ Point Source Catalogue (PSC) data (Beichman et al. 1988; Reipurth et al. 1993) to increase the spectral energy

Send offprint requests to: $\mathrm{R}$. Chini, e-mail: chini@astro.ruhr-uni-bochum.de coverage, and to obtain the temperature of the emitting dust, which we find to be typically $T \leq 30-40 \mathrm{~K}$ (Reipurth et al. 1993; Bontemps et al. 1996; Dent et al. 1998). The temperature can then be used in conjunction with the $\mathrm{mm} / \mathrm{submm}$ fluxes to calculate masses, and hence densities according to Eq. (1) (e.g. Reipurth et al. 1993) by fitting a modified Planck function assuming radiation from a grey-body rather than a black-body. For all calculations, a value for the mass absorption coefficient of $\kappa_{1300}=0.003 \mathrm{~cm}^{2} / \mathrm{g}$ interstellar matter (ISM) was assumed. However, such a fit and therefore any related quantity is strongly dependent on the temperature and also the frequency dependency $\beta$ of the mass absorption coefficient $\kappa_{\nu} \propto \nu^{\beta}$, which can be derived from the submm spectral index.

$M_{\mathrm{gas}}=\frac{S_{\nu} \cdot D^{2}}{\kappa_{\nu} \cdot B_{\nu}\left(T_{\mathrm{dust}}\right)}$.

Consequently, we have obtained submm images at two wavelengths of a number of sources using SCUBA on JCMT; in addition, we have continued our $1300 \mu \mathrm{m}$ survey and detected new sources. In this paper we report on the results of these new $\mathrm{mm} /$ submm observations. 
Table 1. Source positions and flux densities at each wavelength. The positions refer to the $850 \mu \mathrm{m}$ peaks. In each case the peak flux density per beam (peak) is quoted, as well as the integrated flux density (int) within the specified aperture. The peak flux density is defined as the maximum value in the map at the particular position. The integrated flux density only refers to the emission from the source; it was calculated by subtracting the ambient diffuse background emission. The reasons for the choice of the individual aperture sizes are discussed in the text. The integrated flux densities are used in comparison with the IRAS flux densities for fitting the SEDs (see text for details). The statistical errors for each region are listed in Table 2; the absolute calibration uncertainties are $\pm 20 \%$ at $450 \mu \mathrm{m}$ and $\pm 10 \%$ at $850 \mu \mathrm{m}$ and $1300 \mu \mathrm{m}$. The $1300 \mu \mathrm{m}$ entries for the $\mathrm{HH}$ sources were extracted from the original maps of Chini et al. (1997)

\begin{tabular}{|c|c|c|c|c|c|c|c|c|c|}
\hline \multirow[b]{2}{*}{ Source } & \multirow[b]{2}{*}{$\alpha(1950)$} & \multirow[b]{2}{*}{$\delta(1950)$} & \multicolumn{2}{|l|}{$S_{450}$} & \multicolumn{2}{|l|}{$S_{850}$} & \multicolumn{2}{|l|}{$S_{1300}$} & \multirow{2}{*}{$\begin{array}{c}\text { aperture } \\
\text { radius } \\
{\left[{ }^{\prime \prime}\right]}\end{array}$} \\
\hline & & & $\begin{array}{c}\text { peak } \\
{[\mathrm{Jy} / \text { beam }]}\end{array}$ & $\begin{array}{l}\text { int } \\
{[\mathrm{Jy}]}\end{array}$ & $\begin{array}{c}\text { peak } \\
{[\mathrm{Jy} / \text { beam }]}\end{array}$ & $\begin{array}{l}\text { int } \\
{[\mathrm{Jy}]}\end{array}$ & $\begin{array}{c}\text { peak } \\
{[\mathrm{Jy} / \text { beam }]}\end{array}$ & $\begin{array}{l}\text { int } \\
{[\mathrm{Jy}]}\end{array}$ & \\
\hline HH 7-11 MMS 3 & $03^{\mathrm{h}} 25^{\mathrm{m}} 56^{\mathrm{s}} \cdot 6$ & $+31^{\circ} 05^{\prime} 22^{\prime \prime}$ & 4.6 & 10.1 & 1.7 & 0.6 & 0.4 & 0.3 & 10 \\
\hline HH 7-11 MMS 5 & $03^{\mathrm{h}} 25^{\mathrm{m}} 57^{\mathrm{s}} .3$ & $+31^{\circ} 04^{\prime} 53^{\prime \prime}$ & 2.3 & - & 0.8 & - & 0.2 & - & - \\
\hline HH 7-11 MMS 2 & $03^{\mathrm{h}} 25^{\mathrm{m}} 57^{\mathrm{s}} .5$ & $+31^{\circ} 05^{\prime} 36^{\prime \prime}$ & 8.7 & 23.3 & 3.5 & 2.4 & 1.2 & 0.7 & 10 \\
\hline HH 7-11 MMS 1 & $03^{\mathrm{h}} 25^{\mathrm{m}} 58^{\mathrm{s}} .1$ & $+31^{\circ} 05^{\prime} 46^{\prime \prime}$ & 10.7 & 29.8 & 3.4 & 1.8 & 1.3 & 1.0 & 10 \\
\hline HH 7-11 MMS 6 & $03^{\mathrm{h}} 25^{\mathrm{m}} 58^{\mathrm{s}} .5$ & $+31^{\circ} 04^{\prime} 35^{\prime \prime}$ & 1.7 & - & 0.7 & - & 0.1 & - & - \\
\hline HH 7-11 MMS 4 & $03^{\mathrm{h}} 26^{\mathrm{m}} 01^{\mathrm{s}} \cdot 0$ & $+31^{\circ} 05^{\prime} 22^{\prime \prime}$ & 1.5 & 17.8 & 0.9 & 2.8 & 0.2 & 0.7 & 25 \\
\hline HH 7-11 MMS 7 & $03^{\mathrm{h}} 26^{\mathrm{m}} 03^{\mathrm{s}} .2$ & $+31^{\circ} 05^{\prime} 02^{\prime \prime}$ & - & - & 0.9 & - & 0.2 & - & - \\
\hline IRAS $04239+2436$ & $04^{\mathrm{h}} 23^{\mathrm{m}} 54^{\mathrm{s}} .2$ & $+24^{\circ} 36^{\prime} 53^{\prime \prime}$ & 0.9 & 1.2 & 0.3 & 0.2 & 0.1 & 0.1 & 15 \\
\hline L 1527 & $04^{\mathrm{h}} 36^{\mathrm{m}} 49^{\mathrm{s}} \cdot 4$ & $+25^{\circ} 57^{\prime} 21^{\prime \prime}$ & 6.4 & 106.3 & 1.5 & 10.8 & 0.3 & 2.8 & 50 \\
\hline HH 1-2 MMS 3 & $05^{\mathrm{h}} 33^{\mathrm{m}} 52^{\mathrm{s}} .4$ & $-06^{\circ} 47^{\prime} 34^{\prime \prime}$ & 3.4 & 8.0 & 1.1 & 0.5 & 0.2 & 0.1 & 10 \\
\hline HH 1-2 MMS 2 & $05^{\mathrm{h}} 33^{\mathrm{m}} 53^{\mathrm{s}} .0$ & $-06^{\circ} 47^{\prime} 14^{\prime \prime}$ & 4.9 & 9.3 & 1.2 & 0.4 & 0.3 & 0.1 & 8.5 \\
\hline HH 1-2 MMS 1 & $05^{\mathrm{h}} 33^{\mathrm{m}} 57^{\mathrm{s}} \cdot 0$ & $-06^{\circ} 47^{\prime} 56^{\prime \prime}$ & 8.0 & 15.4 & 1.8 & 0.6 & 0.5 & 0.2 & 30 \\
\hline HH 147 MMS & $05^{\mathrm{h}} 33^{\mathrm{m}} 59^{\mathrm{s}} .3$ & $-06^{\circ} 46^{\prime} 28^{\prime \prime}$ & 1.7 & 19.8 & 0.6 & 2.1 & 0.2 & 0.4 & 30 \\
\hline HH 111 MMS & $05^{\mathrm{h}} 49^{\mathrm{m}} 09^{\mathrm{s}} .3$ & $+02^{\circ} 47^{\prime} 46^{\prime \prime}$ & 4.7 & 48.5 & 1.4 & 4.2 & 0.6 & 1.5 & 30 \\
\hline HH 108 IRAS & $18^{\mathrm{h}} 33^{\mathrm{m}} 07^{\mathrm{s}} .8$ & $-00^{\circ} 35^{\prime} 42^{\prime \prime}$ & 4.4 & 24.5 & 0.9 & 2.4 & 0.3 & 0.6 & 25 \\
\hline HH 108 MMS & $18^{\mathrm{h}} 33^{\mathrm{m}} 12^{\mathrm{s}} .1$ & $-00^{\circ} 35^{\prime} 15^{\prime \prime}$ & 2.0 & 9.0 & 0.5 & 1.4 & 0.2 & 0.3 & 25 \\
\hline IRAS 20050+2720 MMS 1 & $20^{\mathrm{h}} 05^{\mathrm{m}} 01^{\mathrm{s}} .9$ & $+27^{\circ} 20^{\prime} 15^{\prime \prime}$ & 13.6 & 37.0 & 2.8 & 3.8 & 0.9 & 1.8 & 20 \\
\hline IRAS $20050+2720$ (incl. outflow) & & & & 184.2 & & 23.9 & & 10.5 & 65 \\
\hline IRAS $20050+2720$ MMS 2 & $20^{\mathrm{h}} 05^{\mathrm{m}} 03.5$ & $+27^{\circ} 18^{\prime} 32^{\prime \prime}$ & - & - & - & - & 0.3 & 0.3 & 15 \\
\hline IRAS $20050+2720$ MMS 3 & $20^{\mathrm{h}} 05^{\mathrm{m}} 06^{\mathrm{s}} .6$ & $+27^{\circ} 18^{\prime} 28^{\prime \prime}$ & - & - & - & - & 0.2 & 0.3 & 15 \\
\hline L 1157 (central source) & $20^{\mathrm{h}} 38^{\mathrm{m}} 39^{\mathrm{s}} .2$ & $+67^{\circ} 51^{\prime} 35^{\prime \prime}$ & 7.9 & 6.0 & 1.5 & 0.9 & 0.4 & 0.4 & 10 \\
\hline L 1157 (source + disk) & & & & 25.3 & & 3.8 & & 1.3 & $55 \times 30$ \\
\hline IRAS $22134+5834$ & $22^{\mathrm{h}} 13^{\mathrm{m}} 24^{\mathrm{s}} \cdot 6$ & $+58^{\circ} 34^{\prime} 12^{\prime \prime}$ & 7.4 & 91.5 & 1.8 & 8.5 & 0.3 & 2.7 & 50 \\
\hline IRAS $23011+6126$ & $23^{\mathrm{h}} 01^{\mathrm{m}} 09^{\mathrm{s}} .7$ & $+61^{\circ} 26^{\prime} 16^{\prime \prime}$ & 12.6 & 43.7 & 1.8 & 4.1 & 0.4 & 1.0 & 40 \\
\hline
\end{tabular}

\section{Observations and data reduction}

Submillimetre continuum observations at $450 \mu \mathrm{m}$ and $850 \mu \mathrm{m}$ were carried out using the Submillimetre Common User Bolometer Array (SCUBA) camera at the James Clerk Maxwell Telescope (JCMT) ${ }^{1}$ on Mauna Kea, Hawaii. The observations were carried out on the evening of 1997 August 6 from 17:30 to 01:30 HST (UT Date: 1997 August 7, 03:30 to 11:30) and the morning of 1997 October 3 from 01:30 to 09:30 HST (UT: 11:30 to 19:30). SCUBA was used in its 64-position jiggling mode to make a fully sampled image of a 2.3 field simultaneously at $450 \mu \mathrm{m}$ and $850 \mu \mathrm{m}$ (Holland et al. 1998). On-source integration

\footnotetext{
1 The James Clerk Maxwell Telescope is operated by the Joint Astronomy Center, Hawaii, on behalf of the UK PPARC, The Netherlands NWO, and the Canadian NRC. SCUBA was built at the Royal Observatory, Edinburgh.
}

times were $10 \mathrm{~s} /$ point in each of the 64 positions, which corresponds to $40 \mathrm{~s} /$ beam at each wavelength.

While the observations were carried out, the secondary mirror was used to chop $150^{\prime \prime}$ in azimuth at around $7 \mathrm{~Hz}$ to reject sky emission. The opacity at both 450 and $850 \mu \mathrm{m}$ was monitored by skydips, and the opacity at $1300 \mu \mathrm{m}$ was monitored by the radiometer located at the adjacent Caltech Submillimetre Observatory. During the August observations the atmospheric opacity was 1.8 at $450 \mu \mathrm{m}$, 0.3 at $850 \mu \mathrm{m}$ and 0.08 at $1300 \mu \mathrm{m}$, typical of average conditions at the site, and corresponding to a zenith atmospheric transmission at $850 \mu \mathrm{m}$ of around $75 \%$. For the October observations the atmospheric opacity was 1.5 at $450 \mu \mathrm{m}, 0.23$ at $850 \mu \mathrm{m}$ and 0.06 at $1300 \mu \mathrm{m}$, typical of above average conditions at the site, and corresponding to a zenith atmospheric transmission at $850 \mu \mathrm{m}$ of around 
$80 \%$. The transmission remained stable throughout both sets of observations.

Pointing and focus were checked using bright sources such as the planet Uranus and the radio source $3 \mathrm{C} 84$, and the pointing was found to be good to $\sim 1^{\prime \prime}$. Calibration was performed using the planets Uranus and Mars (Griffin et al. 1986; Orton et al. 1986; Griffin \& Orton 1993) and the secondary calibration source CRL 618 (Sandell 1994), and a consistent calibration was obtained. We estimate the total absolute calibration uncertainty to be $\sim 20 \%$ at $450 \mu \mathrm{m}$, and $\sim 10 \%$ at $850 \mu \mathrm{m}$. The maps of Uranus show that the JCMT error beam at $450 \mu \mathrm{m}$ is only significant below the $10 \%$ level of peak, and above this the beam is essentially circular. At $850 \mu \mathrm{m}$ the error beam is only significant below the $3 \%$ level. The error beam at $450 \mu \mathrm{m}$ was taken into consideration when calculating the flux densities of our target sources, but at $850 \mu \mathrm{m}$ it was found to be insignificant.

Observations at $1300 \mu \mathrm{m}$ were made with the 37 channel bolometer array at the IRAM $30 \mathrm{~m}$ telescope on Pico Veleta during the winters of 1998 and 1999. The atmospheric opacity as determined from skydips ranged between 0.08 and 0.19 . We used the standard mapping and reduction techniques; for details we refer to one of our earlier papers (e.g. Chini et al. 1997).

We measured the beam-size at each wavelength using the planet Uranus and extra-galactic blazar sources. We found the beam-size to be $8^{\prime \prime} .3$ at $450 \mu \mathrm{m}, 15^{\prime \prime} 0$ at $850 \mu \mathrm{m}$ and $10^{\prime \prime} .7$ at $1300 \mu \mathrm{m}$.

Figure 1 shows our maps of each of the individual regions, which are discussed in detail in Sect. 3 below. Table 1 gives the flux densities of each of the sources we have identified in the maps as well as their peak positions. Table 3 gives the parameters we derived for each of these sources, the details of which are also discussed in Sect. 3. The $1 \sigma$ sky noise in each of the regions was measured from the maps shown in the corresponding figures by calculating the pixel to pixel rms variation of the data. It corresponds to the remaining sky noise in the images after the data reduction and sky subtraction process discussed above; the values are listed in Table 2 .

The peak flux densities in a single beam at each wavelength are listed in Table 1 . We also measured the integrated flux densities of each of the objects in different radius apertures and these are also listed in Table 1. For definition of peak and integrated flux densities see note to this Table. In confused regions where the sources are not fully isolated, it was difficult to apply proper apertures due to the complex structure of the individual fields (multiple and/or elongated sources, diffuse background emission etc.). In some cases we had to choose rather small apertures on the order of the size of the $850 \mu \mathrm{m}$ beam; this may lead to an underestimate of the total flux from these sources. It was necessary to use this smaller aperture on the triple source HH 7-11 MMS 1-3 and the double source HH 1-2 MMS 2-3. All sources were at least marginally resolved except HH 7-11 MMS 1 and 2 at $850 \mu \mathrm{m}$.
Table 2. $1 \sigma$ sky noise of the reduced images, measured from the maps in Fig. 1 in $\mathrm{mJy} /$ beam

\begin{tabular}{lrrr}
\hline Source & $\sigma_{450}$ & $\sigma_{850}$ & $\sigma_{1300}$ \\
& & & \\
\hline HH 7-11 MMS 1-6 & 220 & 180 & 33 \\
IRAS 04239+2436 & 170 & 23 & 4 \\
L 1527 & 400 & 77 & 15 \\
HH 1-2 MMS 1-3 & 440 & 74 & 11 \\
HH 147 MMS & 440 & 74 & 11 \\
HH 111 MMS & 270 & 35 & 24 \\
HH 108 IRAS + MMS & 310 & 37 & 10 \\
IRAS 20050+2720 MMS 1-3 & 370 & 92 & 15 \\
L 1157 & 330 & 28 & 7 \\
IRAS 22134+5834 & 310 & 54 & 16 \\
IRAS 23011+6126 & 410 & 33 & 8 \\
\hline
\end{tabular}

\section{Individual regions}

In this section, we present the maps of the investigated regions and discuss their morphologies. In order to derive the physical properties of the individual sources we have examined their spectral energy distributions (SEDs). This turned out to be complicated since we are comparing lowresolution FIR data with higher resolution $\mathrm{mm} / \mathrm{submm}$ data. First we determined the $1300 \mu \mathrm{m}$ flux densities within the same aperture radii as described above for the submm flux densities; for the $\mathrm{HH}$ regions we derived the corresponding values from the original IRAM data of Chini et al. (1997). Then, where possible, we extracted FIR flux densities from the IRAS PSC (Beichman et al. 1988). In cases where the IRAS PSC values could be designated to multiple sources, we distributed these flux values according to the ratios of their submm flux densities. If there was no IRAS point source, we took the IRAS flux density within the same apertures as used for the submm data from the original IRAS data as upper limits. An exception is HH 108 MMS, for which we have taken ISOPHOT data (Krusch 1999). In combination with the submm SCUBA data, this allowed us to plot the SED for each of our sources from 12 to $1300 \mu \mathrm{m}$. We restrict our fits only to the wavelength range beyond $60 \mu \mathrm{m}$ in order to derive an estimate of the temperature and the mass of the coldest dust component. Due to the crowded nature of some of the regions, however, the SED fitting procedure did not always yield unique solutions. In these cases we discuss the most probable range of parameters. In general, we find that the inferred temperatures $T_{\text {dust }}$ can be constrained to within $\pm 3 \mathrm{~K}$ and the $\beta$-value to about \pm 0.3 , adding another uncertainty to the grey-body fits and therefore a second order uncertainty to the temperature determination. Altogether, this corresponds to a mass indefiniteness of typically a factor of two.

We will define the far-infrared luminosity, $L_{\mathrm{FIR}}$, of each source as the total luminosity of the source longward of $12 \mu \mathrm{m}$. We can measure this by summing the luminosity under the IRAS data points (or upper limits) as far as $100 \mu \mathrm{m}$ plus the luminosity observed by us at 450,850 
Table 3. Table of physical properties as derived from the spectral energy distributions shown in Fig. 2. $L_{\text {FIR }}$ is the total luminosity measured longward of $12 \mu \mathrm{m} ; L_{\mathrm{smm}}$ is the luminosity measured longward of $350 \mu \mathrm{m}$. The molecular hydrogen density $n\left(\mathrm{H}_{2}\right)$ has been determined by assuming a spherical volume of the size of the chosen aperture and the corresponding column density $N\left(\mathrm{H}_{2}\right)$ was calculated based on the covered area

\begin{tabular}{|c|c|c|c|c|c|c|c|c|c|}
\hline Source & $\begin{array}{c}T_{\text {dust }} \\
{[\mathrm{K}]}\end{array}$ & $\beta$ & $\begin{array}{c}\text { Distance } \\
{[\mathrm{pc}]}\end{array}$ & $\begin{array}{l}M_{\text {gas }} \\
{\left[M_{\odot}\right]}\end{array}$ & $\begin{array}{l}L_{\mathrm{FIR}} \\
{\left[L_{\odot}\right]}\end{array}$ & $\begin{array}{c}L_{\mathrm{smm}} \\
{\left[L_{\odot}\right]}\end{array}$ & $\frac{L_{\mathrm{FIR}}}{L_{\mathrm{smm}}}$ & $\begin{array}{l}n\left(\mathrm{H}_{2}\right) \\
{\left[\mathrm{cm}^{-3}\right]}\end{array}$ & $\begin{array}{l}N\left(\mathrm{H}_{2}\right) \\
{\left[\mathrm{cm}^{-2}\right]}\end{array}$ \\
\hline HH 7-11 MMS 1 & 31 & 1.9 & 350 & 2.9 & $<69$ & 0.4 & $<160$ & $3.810^{6}$ & $2.010^{23}$ \\
\hline HH 7-11 MMS 2 & 29 & 1.8 & 350 & 3.0 & $<58$ & 0.4 & $<149$ & $3.910^{6}$ & $2.010^{23}$ \\
\hline HH 7-11 MMS 3 & 30 & 1.8 & 350 & 1.1 & $<23$ & 0.1 & $<177$ & $1.510^{6}$ & $7.710^{22}$ \\
\hline HH 7-11 MMS 4 & 23 & 1.6 & 350 & 4.0 & $<19$ & 0.3 & $<64$ & $3.310^{5}$ & $4.410^{22}$ \\
\hline IRAS $04239+2436$ & 51 & 1.0 & 140 & 0.03 & 1.2 & 0.004 & 288 & $2.010^{5}$ & $6.310^{21}$ \\
\hline L 1527 & 23 & 1.2 & 140 & 3.5 & $<3$ & 0.1 & $<21$ & $5.710^{5}$ & $5.910^{22}$ \\
\hline HH 1-2 MMS 1 & 32 & 2.0 & 460 & 1.4 & $<61$ & 0.2 & $<265$ & $3.010^{4}$ & $6.210^{21}$ \\
\hline HH 1-2 MMS 2 & 40 & 2.0 & 460 & 0.7 & $<29$ & 0.2 & $<182$ & $6.310^{5}$ & $3.710^{22}$ \\
\hline HH 1-2 MMS 3 & 37 & 2.0 & 460 & 0.8 & $<26$ & 0.2 & $<165$ & $4.310^{5}$ & $3.010^{22}$ \\
\hline HH 147 MMS & 26 & 2.0 & 460 & 4.2 & $<64$ & 0.5 & $<121$ & $9.010^{4}$ & $1.910^{22}$ \\
\hline HH 111 MMS & 20 & 1.8 & 460 & 16.6 & 42 & 1.0 & 41 & $3.610^{5}$ & $7.410^{22}$ \\
\hline HH 108 MMS & 13 & 2.0 & 310 & 3.6 & 1 & 0.1 & 11 & $3.310^{5}$ & $5.110^{22}$ \\
\hline HH 108 IRAS & 18 & 2.0 & 310 & 4.5 & $<8$ & 0.3 & $<31$ & $5.410^{5}$ & $6.310^{22}$ \\
\hline IRAS $20050+2720$ MMS 1 & 34 & 1.4 & 700 & 17.1 & 303 & 1.9 & 164 & $3.510^{5}$ & $7.410^{22}$ \\
\hline IRAS $20050+2720$ (incl. outflow) & 28 & 1.0 & 700 & 203 & 375 & 9.2 & 41 & $1.210^{5}$ & $8.310^{22}$ \\
\hline L 1157 (central source) & 29 & 1.2 & 440 & 2.6 & 11 & 0.2 & 63 & $1.710^{6}$ & $1.110^{23}$ \\
\hline L 1157 (source + disk) & 22 & 1.3 & 440 & 14.4 & 15 & 0.6 & 25 & $1.110^{5}$ & $3.810^{22}$ \\
\hline IRAS $22134+5834$ & 45 & 1.0 & 900 & 52.4 & 1518 & 5.5 & 278 & $3.210^{4}$ & $2.210^{22}$ \\
\hline IRAS $23011+6126$ & 27 & 1.5 & 730 & 25.2 & 120 & 1.8 & 67 & $5.710^{4}$ & $2.510^{22}$ \\
\hline
\end{tabular}

and $1300 \mu \mathrm{m}$. The values of $L_{\mathrm{FIR}}$ are listed in Table 3 . We also define each source's submm luminosity, $L_{\mathrm{smm}}$, to be the integrated luminosity longward of $350 \mu \mathrm{m}$. We can then use the ratio of $L_{\mathrm{FIR}} / L_{\mathrm{smm}}$ to attempt to determine the evolutionary status of each source. André et al. (1993) used the total bolometric luminosity, $L_{\mathrm{bol}}$, and defined the protostellar Class 0 stage to be any source that has already formed a central hydrostatic protostar and has $L_{\text {bol }} / L_{\text {smm }} \leq 200$. The more evolved Class I sources all have higher values for this ratio. Since we have data shortward of $12 \mu \mathrm{m}$ only for a few sources, we can generally only use $L_{\mathrm{FIR}} / L_{\mathrm{smm}}$ as a lower limit to the ratio $L_{\mathrm{bol}} / L_{\mathrm{smm}}$. The exact scaling between these two ratios for any particular source depends on the detailed source spectrum. However, the difference between $L_{\mathrm{bol}}$ and $L_{\mathrm{FIR}}$ is - even in extreme cases - in the order of $10 \%$ and thus does not influence our classification significantly.

\section{1. $H H$ 7-11}

Figure 1 shows the new 450 and $850 \mu \mathrm{m}$ images of the region of $\mathrm{HH}$ 7-11 (near SVS 13 in the NGC 1333 complex); the $1300 \mu \mathrm{m}$ map $\left(\mathrm{HPBW} \approx 11^{\prime \prime}\right.$ ) by
Chini et al. (1997) is also included. Due to the similar spatial resolution $\left(7-14^{\prime \prime}\right)$ these images can be compared, and a great deal of similarity is seen. The images show a ridge running roughly from northeast to southwest containing three sources, named MMS 1-3 by Chini et al. (1997). In all three data sets the brightest source is the most northerly, MMS 1, with MMS 2 being the next brightest, and MMS 3 appearing in all three maps as no more than a tail extending to the southeast. However, MMS 3 is seen most clearly in the highest resolution dataset, namely at $450 \mu \mathrm{m}$. The three sources are all closely spaced, and we only just resolve them in our data. HH 7-11 MMS 1 appears to be coincident with a $\mathrm{cm}$ radio source VLA 4, which is a suggested driving source of the HH 7-11 bipolar outflow (Rodríguez et al. 1997). MMS 2 has been recently found to have a cm counterpart, namely VLA 17 (Rodríguez et al. 1999) while MMS 3 is coincident with VLA 2.

Interestingly, the SCUBA images seem to show four additional potential sources. We follow our previous nomenclature and number them by their $\mathrm{mm} / \mathrm{submm}$ strength. The strongest of them lies east of the ridge just described. This source is also visible in the $1300 \mu \mathrm{m}$ image 

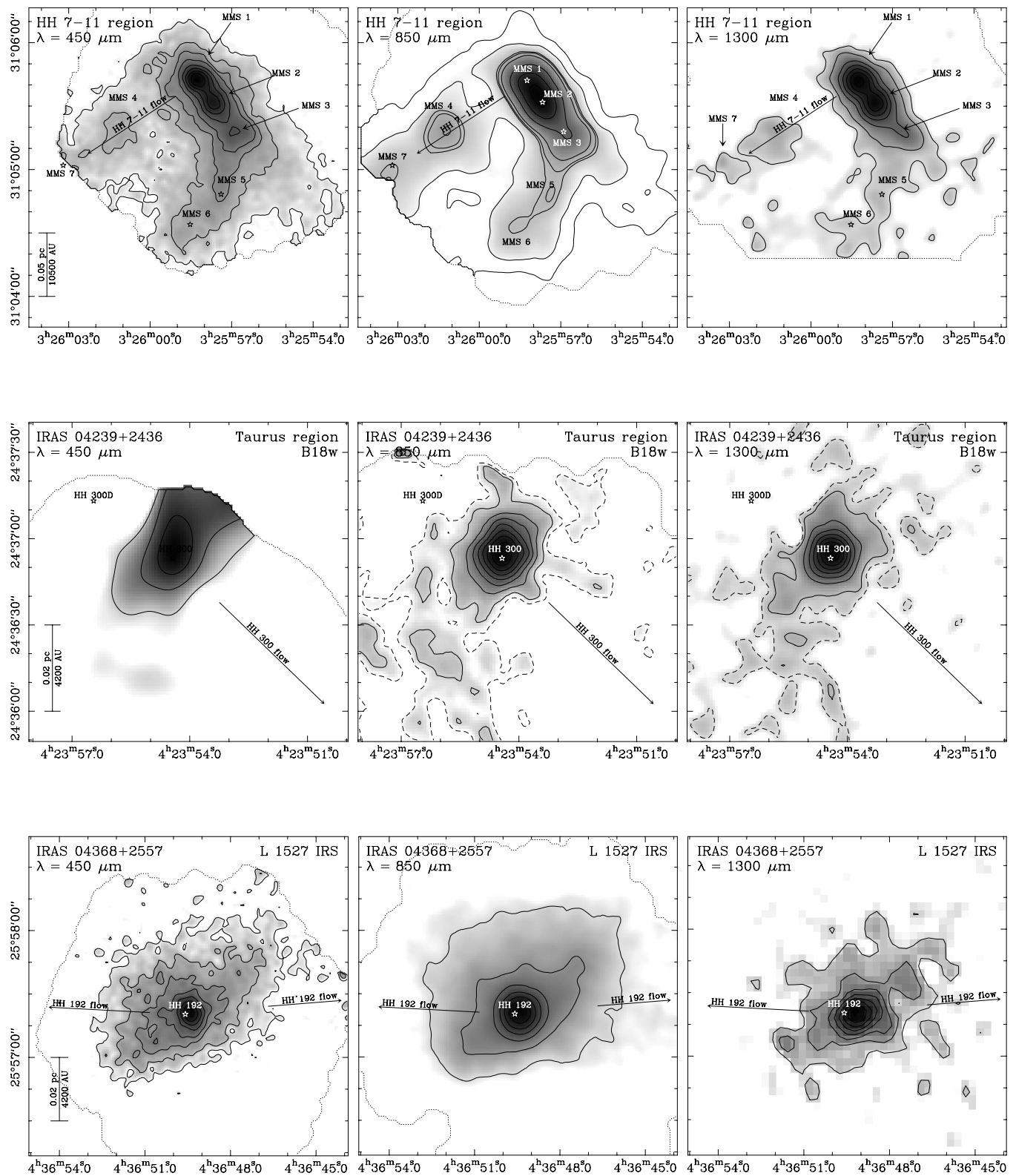

Fig. 1. Grey scale and contour plots of the investigated regions. Table 2 contains the values of $\sigma$ in each field (see also text for discussion). All axes are marked in epoch 1950. HH 7-11 region (upper row): $450 \mu \mathrm{m}$, contours at $3 \sigma, 6 \sigma, 9 \sigma, 12 \sigma$, then steps of $6 \sigma$ upwards; $850 \mu \mathrm{m}$, contours at $3 \sigma, 5 \sigma, 7.8 \sigma, 8.4 \sigma, 10 \sigma, 16.5 \sigma$, then steps of $8 \sigma$ upwards; $1300 \mu \mathrm{m}$, contours at $3 \sigma, 6 \sigma$, then steps of $6 \sigma$ upwards. IRAS 04239+2436 (middle row): $450 \mu \mathrm{m}$ (smoothed to resolution of $850 \mu \mathrm{m}$ ) and $850 \mu \mathrm{m}$, contours from $3 \sigma$ upwards in steps of $2 \sigma ; 1300 \mu \mathrm{m}$, contours from $3 \sigma$ upwards in steps of $3 \sigma$; dashed contours represent a level of $2 \sigma$. L 1527 (lower row): $450 \mu \mathrm{m}$, contours at $3 \sigma, 5 \sigma, 7 \sigma, 9 \sigma$ and then upwards by $3 \sigma ; 850 \mu \mathrm{m}$, contours from $3 \sigma$ upwards in steps of $3 \sigma ; 1300 \mu \mathrm{m}$, contours from $3 \sigma$ upwards in steps of $2 \sigma$

of Chini et al. (1997) but was not recognized as an individual source there due to its low signal-to-noise ratio. However, the presence of the source at all three wavebands leads us to conclude that it is real; we here name it HH 7-11 MMS 4. We note that this source also appears in the submm data of Chandler \& Richer (2000) although these authors did not remark upon it. Its position coincides with the Herbig-Haro object HH 8 (see e.g. Herbig \& Jones 1983; Chrysostomou et al. 2000) and with faint CO emission observed by Grossman et al. (1987). A further tentative source appears at the eastern edge of the 450 and $850 \mu \mathrm{m}$ maps which also is indicated on our original $1300 \mu \mathrm{m}$ images. This source - if real - would coincide with the position of $\mathrm{HH} 7$; we here name this source HH 7-11 MMS 7. Accurate photometry of this source cannot be established due to its position at the edge of the maps. Therefore, it has been excluded from Tables 1 and 3. A stellar origin of MMS 4 and MMS 7 can be ruled out, as Aspin et al. (1994) classified them as nebulous near-infrared sources ASR 6 and ASR 22, matching $1.1 \mathrm{~mm}$ peaks (see Fig. 7 in their paper) similar to our maps. Additionally, neither source corresponds to 

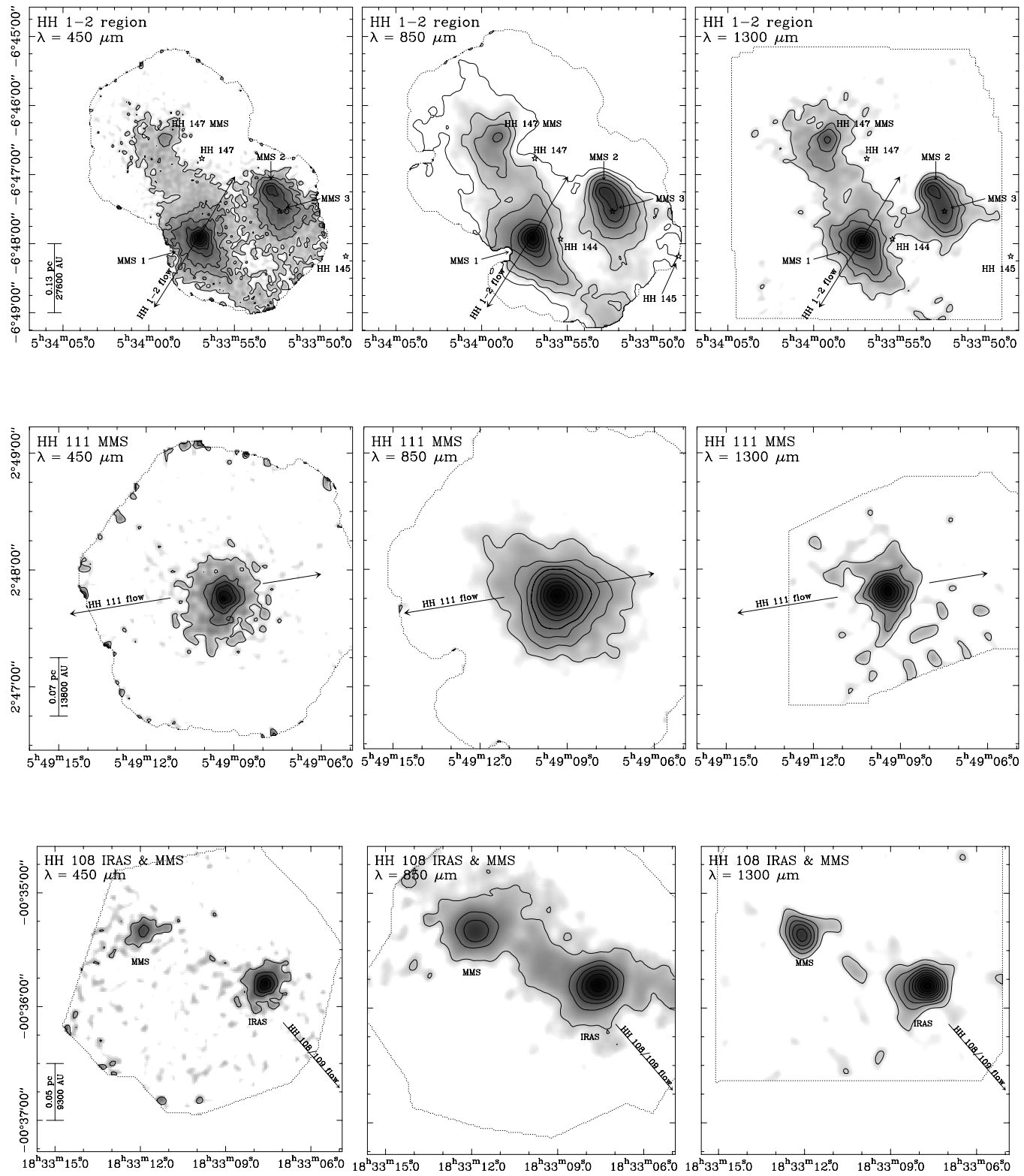

Fig. 1. continued. HH 1-2 / HH 147 region (upper row): $450 \mu \mathrm{m}$, contours at $3 \sigma, 6 \sigma, 9 \sigma$, then steps of $6 \sigma$ upwards; $850 \mu \mathrm{m}$, contours at $3 \sigma, 6 \sigma, 9 \sigma$, then steps of $6 \sigma$ upwards; $1300 \mu \mathrm{m}$, contours at $3 \sigma, 6 \sigma, 9 \sigma, 15 \sigma, 20.5 \sigma, 27 \sigma$, then steps of $6 \sigma$ upwards. HH 111 region (middle row): $450 \mu \mathrm{m}$, contours from $3 \sigma$ upwards in steps of $4 \sigma ; 850 \mu \mathrm{m}$, contours from $3 \sigma$ to $12 \sigma$ in steps of $3 \sigma$ and then upwards in steps of $6 \sigma ; 1300 \mu \mathrm{m}$, contours from $3 \sigma$ upwards in steps of $3 \sigma$. HH 108 region (lower row): $450 \mu \mathrm{m}$, contours from $3 \sigma$ upwards in steps of $6 \sigma ; 850 \mu \mathrm{m}$, contours from $3 \sigma$ upwards in steps of $6 \sigma$; $1300 \mu \mathrm{m}$, contours from $3 \sigma$ upwards in steps of $3 \sigma$

any of the VLA sources found by Rodríguez et al. (1999). This might be indicating that we are seeing - for the first time - HH objects directly at $\mathrm{mm} /$ submm wavelengths, in which case the emission would probably originate from density enhancements of the dusty environment produced by the interacting $\mathrm{HH}$ jet. Although it is generally considered that most of the pre-shock material evaporates during interaction with the outflow, Mouri \& Taniguchi (2000) recently showed that $70-80 \%$ of the grains could escape destruction. It is noteworthy that Molinari et al. (1999) report on the detection of crystalline water ice in HH 7 which has formed on dust grains. Their production can only be explained with grain temperatures $\gtrsim 100 \mathrm{~K}$ which can be attained in dissociative shocks. This would imply a large amount of post-shock dust in this area.

Two other new mm sources appear south of the main ridge in the 450 and $850 \mu \mathrm{m}$ images. Again there are weak indications for their presence in our earlier $1300 \mu \mathrm{m}$ data. Within the positional uncertainties, the stronger one, HH 7-11 MMS 5, is coincident with VLA 16, and the fainter one, HH 7-11 MMS 6, with VLA 19. Both of them are identified as YSOs by Rodríguez et al. (1999). VLA 16 is a time variable source exhibiting large circular polarization and coincides with the NIR source ASR 7 (Aspin et al. 1994), which is believed to be a T Tauri star. VLA 19 exhibits a spectral index at $\mathrm{cm}$ wavelengths which 

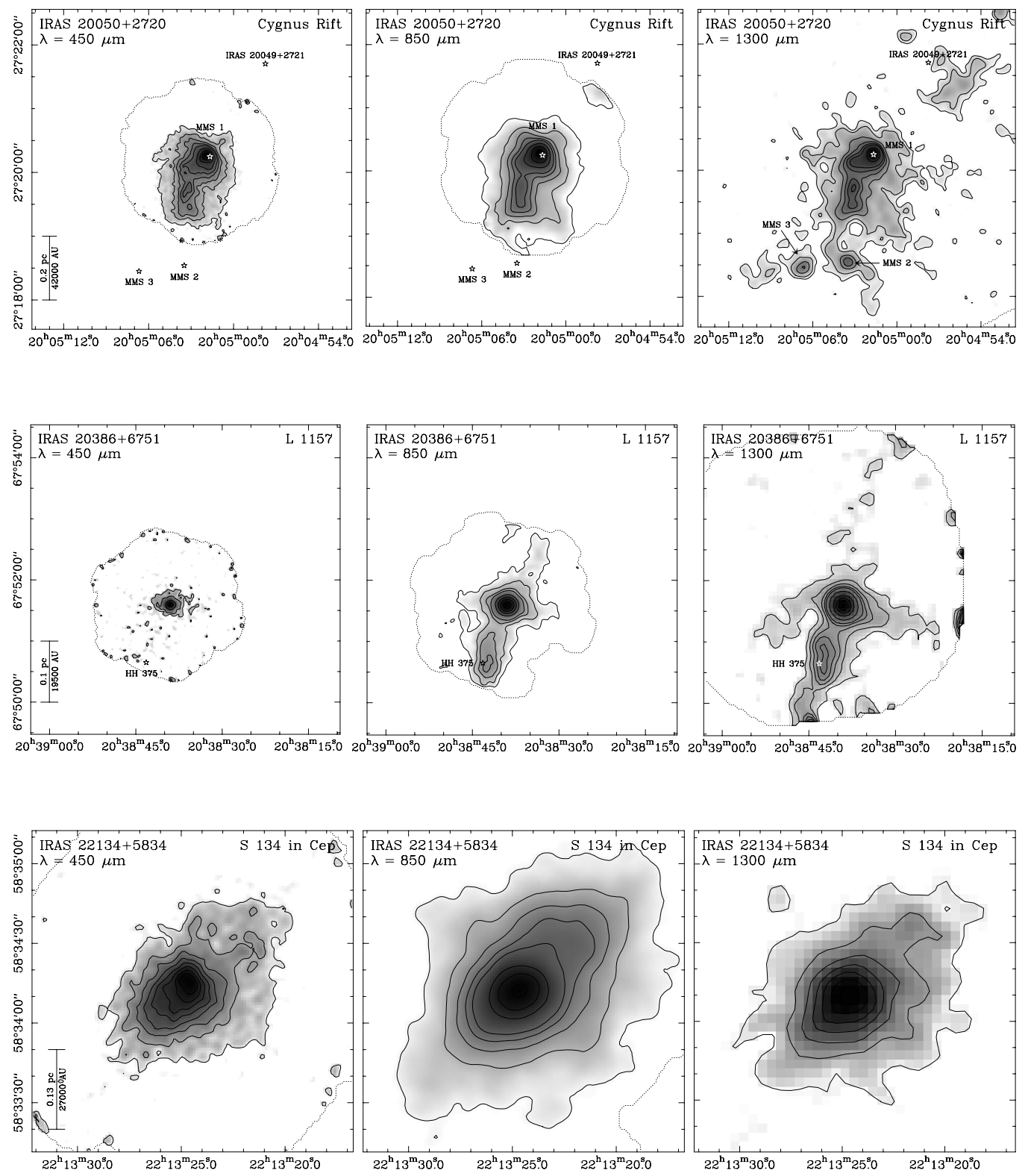

Fig. 1. continued. IRAS 20050+2720 (upper row): $450 \mu \mathrm{m}$ and $850 \mu \mathrm{m}$, contours from $3 \sigma$ to $15 \sigma$ in steps of $3 \sigma$ and then from $21 \sigma$ in steps of $7 \sigma ; 1300 \mu \mathrm{m}$, contours at $3 \sigma, 5 \sigma$ to $30 \sigma$ in steps of $5 \sigma$ and then in steps of $10 \sigma$. L 1157 (middle row): $450 \mu \mathrm{m}$ with contours at $3 \sigma, 6 \sigma$ and then upwards in steps of $10 \sigma ; 850 \mu \mathrm{m}$ with contours from $3 \sigma$ to $12 \sigma$ in steps of $3 \sigma$ and then in steps of $11 \sigma ; 1300 \mu \mathrm{m}$ with contours from $3 \sigma$ to $15 \sigma$ in steps of $3 \sigma$ and from $25 \sigma$ upwards in steps of $20 \sigma$. IRAS 22134+5834 (lower row): $450 \mu \mathrm{m}$ with contours from $3 \sigma$ upwards in steps of $3 \sigma ; 850 \mu \mathrm{m}$ with contours from $3 \sigma$ to $18 \sigma$ in steps of $3 \sigma$ and then upwards by $6 \sigma ; 1300 \mu \mathrm{m}$ with contours from $3 \sigma$ upwards in steps of $3 \sigma$

also suggests an association with a young star. Thus, it is very likely that we have detected the $\mathrm{mm} / \mathrm{submm}$ counterparts of two further young objects in the $\mathrm{HH}$ 7-11 region.

Figure 2 displays the SEDs of HH 7-11 MMS 1-4. In order to avoid overlapping apertures the submillimetre fluxes for MMS 1-3 were measured in a radius of $10^{\prime \prime}$. The remaining source, MMS 4, was measured in a $25^{\prime \prime}$ radius aperture (excluding the segment containing the source MMS 7). IRAS only detected a single point source in the area that coincides with the strong $\mathrm{mm} / \mathrm{submm}$ ridge. Consequently, we do not know which of the three submm sources is the IRAS source, or whether all three contribute at some level. Therefore, we applied the procedure of distributing the $I R A S$ flux density according to the ratio of the submm flux density.

MMS 1-3 all have central protostellar sources, as evinced by the presence of $\mathrm{cm}$ radio continuum emission. The value of the $L_{\mathrm{FIR}} / L_{\mathrm{smm}}$ ratio for each source is listed in Table 3. By adding the NIR photometry values from Aspin et al. (1994) to our data, we can extend the spectral coverage for MMS 1 and 4, which are listed there as sources ASR 1 and ASR 6. This allows us to derive bolometric luminosities of $L_{\mathrm{bol}}=81 L_{\odot}$ for MMS 1 and $<23 L_{\odot}$ for MMS 4 . The resulting $L_{\mathrm{bol}} / L_{\mathrm{smm}}$ ratios 


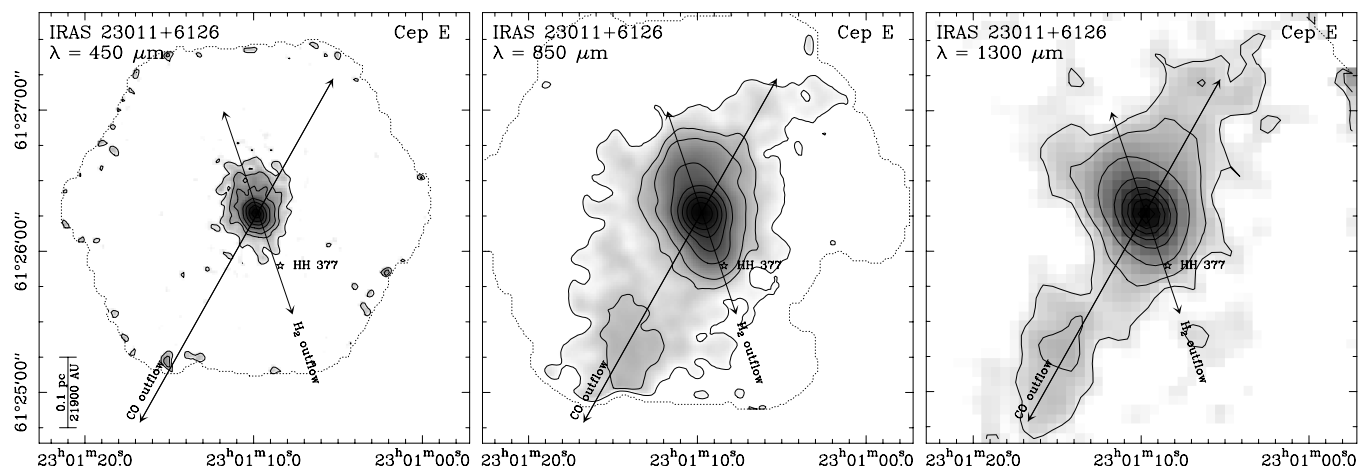

Fig. 1. continued. IRAS 23011+6126: $450 \mu \mathrm{m}$ with contours at $3 \sigma, 6 \sigma, 9 \sigma$ and then upwards in steps of $6 \sigma ; 850 \mu \mathrm{m}$ with contours at $3 \sigma, 6 \sigma, 9 \sigma$ and then from $19 \sigma$ in steps of $10 \sigma ; 1300 \mu \mathrm{m}$ with contours at $3 \sigma, 5 \sigma$ and then from $10 \sigma$ upwards in steps of $11 \sigma$
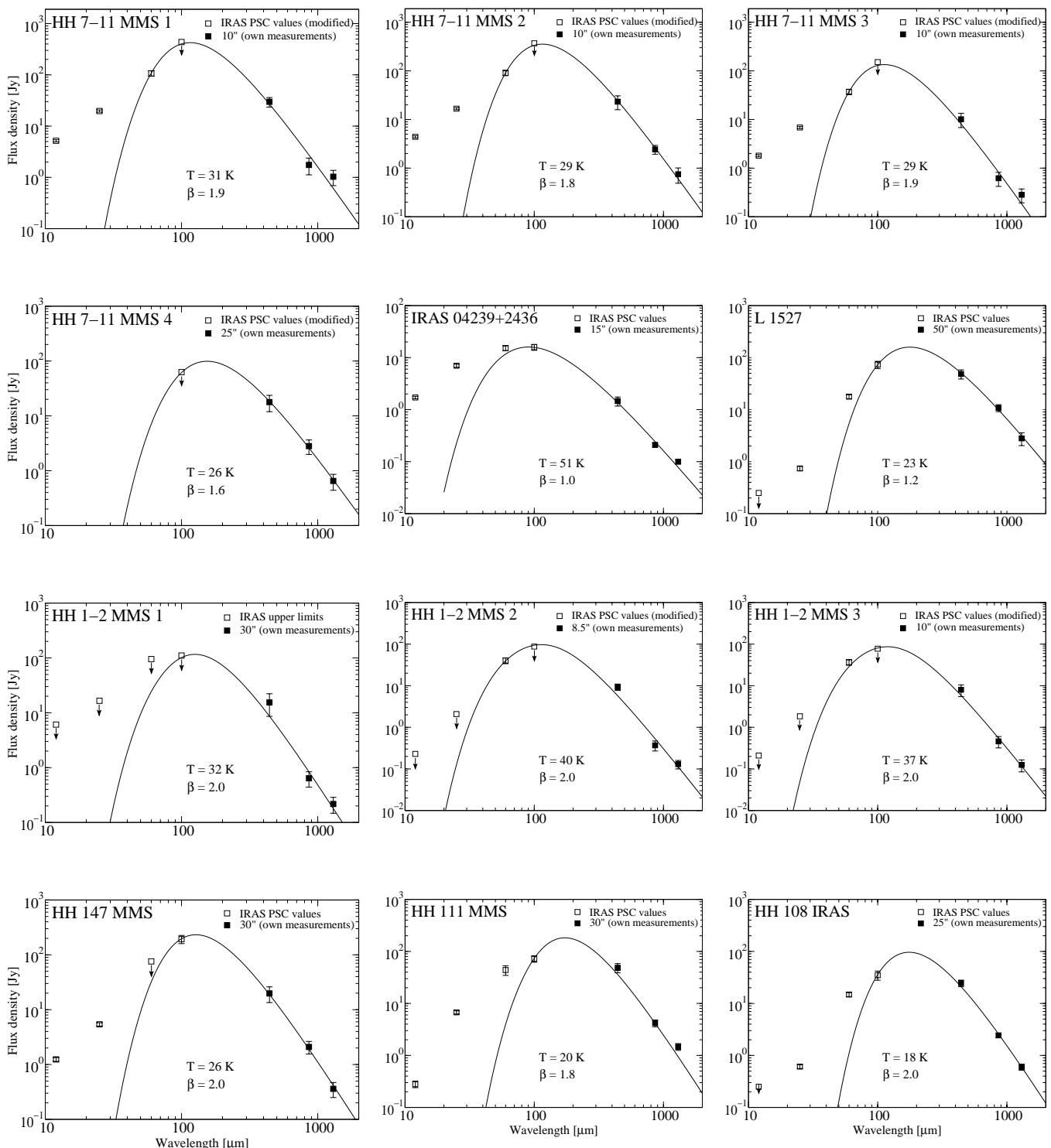

Fig. 2. Spectral energy distributions of the individual sources. The FIR fluxes are taken from the IRAS PSC. The solid lines are fitted modified Planck functions (grey-bodies). We have also labelled the plot with the fitted temperature and the submm-slope $\beta$

are 189 for MMS 1 and <77 for MMS 4. Obviously all 200, although being close to the borderline. The fact that 3 sources MMS 1-3 have values of $L_{\mathrm{FIR}} / L_{\mathrm{smm}}$ less than Gregersen et al. (2000) did not find any evidence for infall 

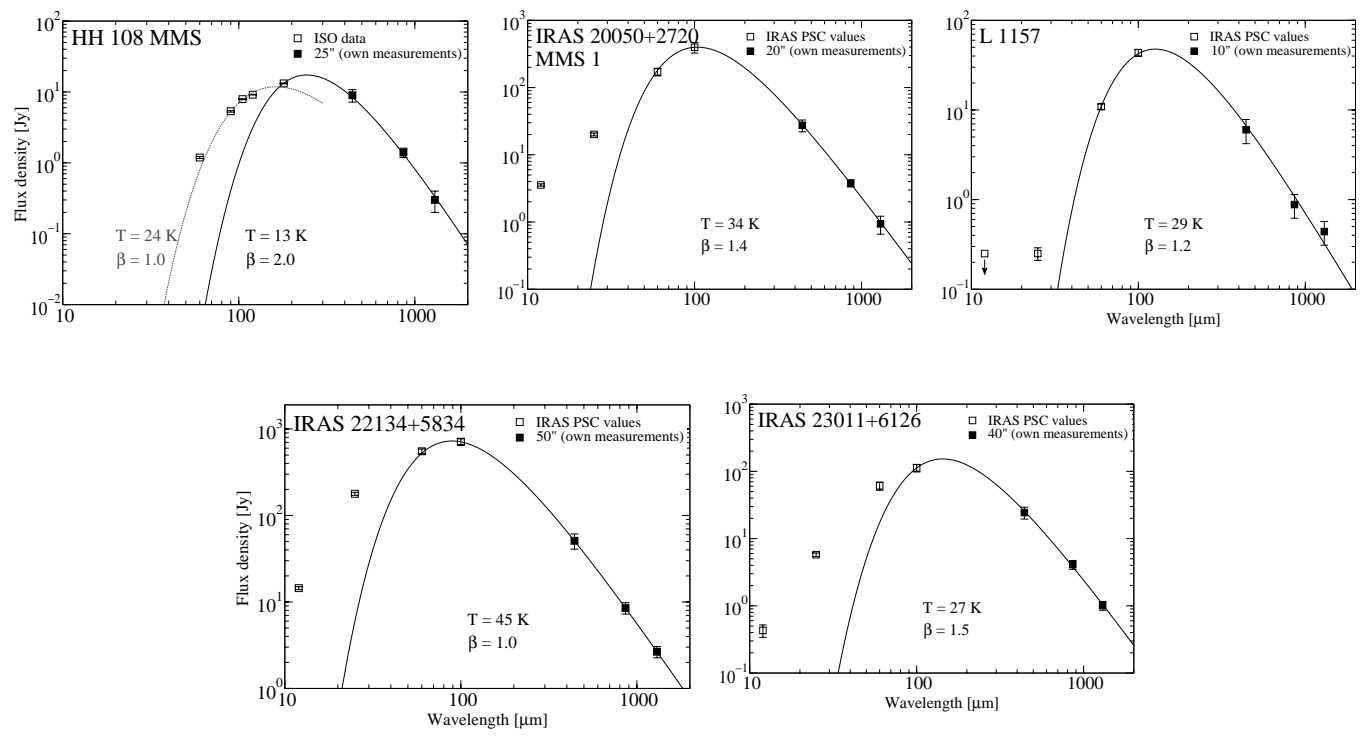

Fig. 2. continued

of circumstellar matter related to MMS 1 makes a Class I nature very likely. In summary, in the region of HH $7-$ 11 we have detected: three young stellar objects (YSOs), MMS 1-3; two HH objects, MMS 4 and 7; and two T Tauri stars, MMS 5 and 6 . If our interpretation is correct, this corresponds to the first direct $\mathrm{mm} / \mathrm{submm}$ continuum detections of $\mathrm{HH}$ objects themselves.

\subsection{IRAS $04239+2436$}

IRAS $04239+2436$ lies within the Taurus molecular cloud region at an approximate distance of $140 \mathrm{pc}$ (Kenyon et al. 1994). It drives the HH 300 giant bipolar outflow jet (Reipurth et al. 1997) and is located in a small core detected in $\mathrm{C}^{18} \mathrm{O}$ by Onishi et al. (1998) - their source No. 13. The core appears to have a flattened high density CS envelope perpendicular to the outflow axis (Ohashi et al. 1996). It was detected in $\mathrm{cm}$ radio continuum with the VLA by Rodriguez \& Reipurth (1998), indicating the presence of a central young stellar object (YSO) driving the outflowing jet. The YSO has a peculiar infrared spectrum (Greene \& Lada 1996). Recent HST NICMOS images show that it is a close pre-main-sequence (PMS) binary (Reipurth et al. 2000).

Figure 1 shows the 450, 850 and $1300 \mu \mathrm{m}$ images of the IRAS $04239+2436$ region. The 450 and $1300 \mu \mathrm{m}$ data have been smoothed to the resolution of the $850 \mu \mathrm{m}$ data. All three images show a very similar structure - basically a single, marginally resolved source at this resolution. We measured the peak flux density at each wavelength, and list this in Table 1, as well as the integrated flux density in a $15^{\prime \prime}$ radius circular aperture, since this encompassed all of the flux above the $3 \sigma$ level (see Table 2). The fact that the integrated flux densities are all similar to the peak flux density shows that this is most likely a point source with some diffuse extended circumstellar matter as outlined by the $2 \sigma$ contours.
Figure 2 shows the spectral energy distribution of this source, wherein we have used the integrated flux densities from Table 1, combined with the IRAS PSC flux densities, since even a source with radius $15^{\prime \prime}$ would appear as a point source to IRAS. The 100-1300 $\mu \mathrm{m}$ data can all be fitted by a single temperature modified blackbody function of temperature $51 \mathrm{~K}$ and $\beta=1$.0. The shorter wavelength IRAS data require additional hotter dust components to explain their flux densities. NIR and MIR photometry by Myers et al. (1987) yields a total luminosity of $1.2 L_{\odot}$ shortward of $100 \mu \mathrm{m}$. The source is not visible at optical wavelengths (Tamura et al. 1991).

As $L_{\text {FIR }}$ underestimates the total luminosity of this source, we added the NIR measurements of Myers et al. (1987) to our calculations. This gives $L_{\mathrm{bol}}=$ $1.48 L_{\odot}$ resulting in a $L_{\mathrm{bol}} / L_{\mathrm{smm}}$ ratio of 370 . Therefore, IRAS $04239+2436$ is definitely the most evolved source in our present sample and has to be regarded as a Class I source.

\section{3. $L 1527$}

L 1527 is a cloud within the Taurus molecular cloud complex, at a distance of $140 \mathrm{pc}$ (Kenyon et al. 1994). The embedded IRAS source IRAS $04368+2557$ drives a compact molecular outflow (Tamura et al. 1996). While it was detected with the VLA (Rodriguez \& Reipurth 1998) only a nebula is seen in the NIR (Tamura et al. 1996). Interferometry measurements are discussed by Ohashi et al. (1997) and Zhou et al. (1996), who detected protostellar infall towards L 1527, which is generally regarded as an indicator for extremely young protostellar sources. Similar results have been reported by Maradones et al. (1997) and Gregersen et al. (2000). Interferometric millimetre observations were carried out by Hogerheijde et al. $(1997,1998)$. These authors quote line intensities of molecular transitions (e.g. ${ }^{12} \mathrm{CO}(3-2),{ }^{12} \mathrm{CO}(6-5)$ ), 
which could contaminate the continuum flux density measurements at 450 and $850 \mu \mathrm{m}$. However, a closer examination led to the conclusion that line contribution is negligible in these cases $(<1 \%)$.

Figure 1 shows the 450, 850 and $1300 \mu \mathrm{m}$ images of the $\mathrm{L} 1527$ region at the full spatial resolution at which they were observed. The images all show a very similar morphology, consisting of a compact source at the centre, surrounded by more extended, lower level emission. We interpret this as an enshrouded central source surrounded by a dust envelope that is elongated in a $\mathrm{SE}-\mathrm{NW}$ direction. Using the above assumed distance it has a semimajor axis of $5300 \mathrm{AU}$ and a semi-minor axis of $2400 \mathrm{AU}$ at the $5 \sigma$ level, and an axial ratio of $2.2: 1$. The long axis of the object that we see is in the same orientation as the source extension seen in interferometric millimetre transitions (Hogerheijde et al. 1997, 1998).

Eiroa et al. (1994) associated HH emission with L 1527 and concluded that the outflow was driven by a Class 0 source with an edge-on disk. Later observations of this HH 192 outflow (Gomez et al. 1997) showed an orientation that is roughly east-west. Hence the outflow is not aligned with either the major or minor axis of our $\mathrm{mm} / \mathrm{submm}$ images, nor with the mm interferometer source extension. Our value for $L_{\mathrm{FIR}} / L_{\mathrm{smm}}<21$ corroborates the Class 0 nature of this source.

Table 1 shows the peak flux densities, as well as the integrated flux densities in a $50^{\prime \prime}$ radius circular aperture centred on this source. This was chosen as it encompasses all of the flux above $3 \sigma$ in this region. These flux densities were plotted along with the IRAS PSC flux densities in Fig. 2. This source can be seen to be significantly brighter than the previous source at FIR and $\mathrm{mm} / \mathrm{submm}$ wavelengths, despite being at roughly the same distance. However, it also contains a significant amount of colder dust, since its 100-1300 $\mu \mathrm{m}$ emission can be fitted with a single grey-body with $T_{\text {dust }}=23 \mathrm{~K}$ and $\beta=1.2$. There is also less evidence for hotter dust in this case since the source was not detected by IRAS at $12 \mu \mathrm{m}$.

\section{4. $H H 1-2, H H 147$}

Figure 1 shows the 450 and $850 \mu \mathrm{m}$ images of the region around HH 1-2 MMS 1-3 and HH 147 MMS. Once again the similarity with the $1300 \mu \mathrm{m}$ map by Chini et al. (1997) is apparent. The HH 1-2 / HH 147 region shows three separate clumps of emission. The north-easterly source is the source we previously named HH 147 MMS. The source to the south-east we named HH 1-2 MMS 1 (see Chini et al. 1997). The clump to the west contains two separate sources, which we named HH 1-2 MMS 2 and 3 respectively. All of these sources can be seen in both the 450 and $850 \mu \mathrm{m}$ data, although MMS 2 and 3 are only barely resolved at $850 \mu \mathrm{m}$.

The slightly extended source HH 1-2 MMS 1 contains within its contours two cm radio sources VLA 1 and 2. VLA 1 has been previously identified as the driving source of the outflow with which HH 1 and 2 are associated (Pravdo et al. 1985; Rodríguez et al. 1990). Further to the north-west there is an optically visible T Tauri star (Cohen \& Schwartz 1979). An overview of the region is given by Reipurth et al. (1993). We noted in our previous paper (Chini et al. 1997) that the elongation of MMS 1 is roughly perpendicular to the direction of the $\mathrm{HH}$ 1-2 outflow, and we see the same elongation here at both 450 and $850 \mu \mathrm{m}$. We note that this is also parallel to the elongation observed in $\mathrm{H}^{13} \mathrm{CO}^{+}$(Choi \& Lee 1998). HH 12 MMS 2 and 3 are also in a slightly confused region, containing VLA cm sources and masers (see discussion in Chini et al. 1997). HH 147 MMS is coincident with a T Tauri star, source No. 3 of Strom et al. (1985), which drives the HH 147 bipolar outflow (Eislöffel et al. 1994).

Figure 2 displays the SEDs of $\mathrm{HH} 1-2$ MMS 1-3 and HH 147 MMS. The IRAS point source IRAS 05339-0646 coincides with our position of $\mathrm{HH} 147 \mathrm{MMS}$, while the source IRAS 05338-0647 coincides with the ridge that contains HH 1-2 MMS 2-3. It is unclear which of the two millimetre sources corresponds to IRAS 05338-0647, therefore we have divided its flux density according to the ratio of the submillimetre flux densities of the sources. An aperture with a radius of $30^{\prime \prime}$ was used for HH 147 MMS and HH 1-2 MMS 1. Due to source confusion it was necessary to use an aperture of radius $8 . .5$ for MMS 2 and $10^{\prime \prime}$ for MMS 3. This probably leads to an underestimate of the $850 \mu \mathrm{m}$ fluxes. The calculation of the dust temperature for MMS 1 is limited by the fact that IRAS PSC values do not exist. The derived upper limits of the FIR flux densities (see head of Sect. 3) lead to a temperature of $32 \mathrm{~K}$.

We can also compare the ratios of $L_{\mathrm{FIR}} / L_{\mathrm{smm}}$ for each source. Treating MMS 1 as a single source it does not satisfy the Class 0 criterion. However, as it contains more than one VLA source it is clearly a multiple object and thus this classification criterion might be problematic. Because MMS 2 and 3 are only partially resolved in our data and MMS 2 contains at least two known VLA sources, their appearance as Class 0 objects must also be treated with caution. Finally, HH 147 MMS exhibits a ratio $L_{\mathrm{FIR}} / L_{\mathrm{smm}}$ of about 120 indicating a Class 0 source. Including the photometry shortward of $12 \mu \mathrm{m}$ (Strom et al. 1985), the bolometric luminosity $L_{\text {bol }}$ rises up to $81 L_{\odot}$ which shifts the source close to the transition between Class 0 and I with a ratio $L_{\mathrm{bol}} / L_{\mathrm{smm}}$ of 189 . This result can be explained by the following two scenarios: either we measure the emission of circumstellar disk around a T Tauri star, or we are dealing with two separate sources of which the visible $\mathrm{T}$ Tauri star may have been ejected from the cloud core that we have detected at submm/mm wavelengths.

\section{5. $H H 111$}

Figure 1 shows the 450 and $850 \mu \mathrm{m}$ images of the region of HH 111 MMS and the very similar $1300 \mu \mathrm{m}$ map of 
Chini et al. (1997). A single source is seen, with a faint extension to the south. This is the driving source of the HH 111 bipolar outflow (Reipurth 1989). The source is barely resolved at $850 \mu \mathrm{m}$, but can be seen to be extended in the higher resolution $450 \mu \mathrm{m}$ data. However, at this wavelength the telescope error beam confuses any interpretation of the low level extended structure. HH 111 is coincident with the IRAS source IRAS $05491+0247$ which provides an FIR luminosity of $25 L_{\odot}$ (Reipurth 1989). With observations at longer wavelengths we derive a total FIR luminosity of $42 L_{\odot}$. Figure 2 displays the SED of HH 111 as measured with an aperture of radius $30^{\prime \prime}$. The ratio of $L_{\mathrm{FIR}} / L_{\mathrm{smm}}$ is less than 200 in this case. However, the IRAS detections at all four wavebands indicate that there is more emission shortward of $12 \mu \mathrm{m}$, thus $L_{\mathrm{FIR}}$ is clearly underestimating $L_{\text {bol }}$ in this source. In addition, new infrared observations with the HST and the VLA suggest that we may be dealing with a young triple system (Reipurth et al. 1999) which prevents any unique classification.

\section{6. $H H 108$}

Figure 1 shows the region around HH 108 MMS and HH 108 IRAS at 450, 850 and $1300 \mu \mathrm{m}$. The latter is taken from Chini et al. (1997), and once again a very similar morphology is seen at all three wavelengths. In addition we observe what appears to be a bridge of emission linking the two sources. This is seen most clearly at $850 \mu \mathrm{m}$, but it also appears faintly in each of the other two images, so we believe that this is a real feature. HH 108 IRAS is the likely driving source of the HH 108-109 bipolar outflow (Reipurth \& Eiroa 1992). However, HH 108 MMS was a newly discovered source by Chini et al. (1997), who speculated that it might be a similar source to HH 24 MMS (Chini et al. 1993) or HH 114 MMS (Chini et al. 1997). We discuss its nature below.

Figure 2 shows the SEDs of $\mathrm{HH} 108$ MMS and HH 108 IRAS, which coincides with the IRAS source IRAS 18331-0035. IRAS fluxes are not available for HH 108 MMS, but luckily we can make use of recent ISOPHOT data (Krusch 1999). The fluxes were measured in $25^{\prime \prime}$ radius apertures and the contribution from the ridge was removed. As derived from grey-body fits, both sources seem to be the coldest in our sample yielding $18 \mathrm{~K}$ for the IRAS source and $13 \mathrm{~K}$ for HH $108 \mathrm{MMS}$, which appears to possess at least a second dust component of $24 \mathrm{~K}$. Due to the fact that the IRAS flux value for HH 108 IRAS at $12 \mu \mathrm{m}$ is only an upper limit, $L_{\text {FIR }}$ should be a good estimate for $L_{\mathrm{bol}}$. Therefore, all our results classify it as a Class 0 source. However, Gregersen et al. (2000) could not find any evidence for matter infall.

HH 108 MMS does not appear to have been detected other than in the FIR or mm/submm; its $L_{\mathrm{FIR}} / L_{\mathrm{smm}}$ ratio of 11 suggests that it is potentially another similar source to HH 24 MMS (Chini et al. 1993; Ward-Thompson et al. 1995), namely an extremely young protostellar source, either on the verge of collapse or just beginning the protostellar collapse phase. This source would bear further investigation at other wavelengths.

\subsection{IRAS $20050+2720$}

IRAS $20050+2720$ is somewhat different from some of the other sources. It is a medium-mass object with a luminosity of $388 L_{\odot}$ (Molinari et al. 1996) surrounded by a large cluster of up to 100 low-mass stars (Chen et al. 1997; di Francesco \& Evans 1998) and located in the Cygnus rift at a distance of around $700 \mathrm{pc}$ (Bachiller et al. 1995). Extended $2.7 \mathrm{~mm}$ continuum emission was detected by Wilking et al. (1989), and a very high velocity multipolar outflow by Bachiller et al. (1995) and Codella et al. (1999). Millimetre observations were made by Choi et al. (1999) and Gregersen et al. (1997) who found blue-skewed molecular line emission indicative of infalling gas. However, they deduced that the region is too complex to be modelled in terms of spherically symmetric collapse.

Figure 1 shows the 450, 850 and $1300 \mu \mathrm{m}$ images of the IRAS $20050+2720$ region. A bright central object is seen at all wavelengths, together with an extension to the south. Additional sources are seen in the somewhat larger $1300 \mu \mathrm{m}$ image, which are outside of the area mapped by SCUBA. According to our former procedure we name the bright central object coinciding with IRAS $20050+2720$ as MMS 1. The other two sources to the south, which are probably detected for the first time, are called MMS 2 and 3 and their $1300 \mu \mathrm{m}$ flux densities are listed in Table 1 as well as the other results of the photometry carried out on this object. In order to derive properties only for MMS 1 we first performed flux density measurements within an aperture of $20^{\prime \prime}$ radius. The results are shown Table 3 , between them $T_{\text {dust }}=34 \mathrm{~K}, \beta=1.4$ and $M_{\text {gas }}=17.1 M_{\odot}$. If we extend the aperture up to $65^{\prime \prime}$ radius covering also the prominent extent to the south which seems also more comparable to the less resolved IRAS PSC measurements, we derive $T_{\text {dust }}=28 \mathrm{~K}, \beta=1.0$ and $M_{\text {gas }}=203 M_{\odot}$. Together with the obtained luminosities from Table 3, we think it is safe to conclude that most of the luminosity is produced in the highly populated star cluster around MMS 1. Yet, most of the interstellar matter seems to be located in the southern $\sim 20000$ AU long extended structure. At this distance and within the crowded cluster field, there may be confusion by several sources in our beam. Taking the results of MMS 1 alone, it is the second most massive and luminous object in our sample. This is clearly a young cluster in the process of formation, and we may be seeing the start of a sequence of rich star formation in this area.

\section{8. $L 1157$}

L 1157 is located in a small isolated cloud in Cepheus, at an adopted distance of 440 pc (Viotti 1969). The embedded FIR source IRAS $20386+6751$ is a single 

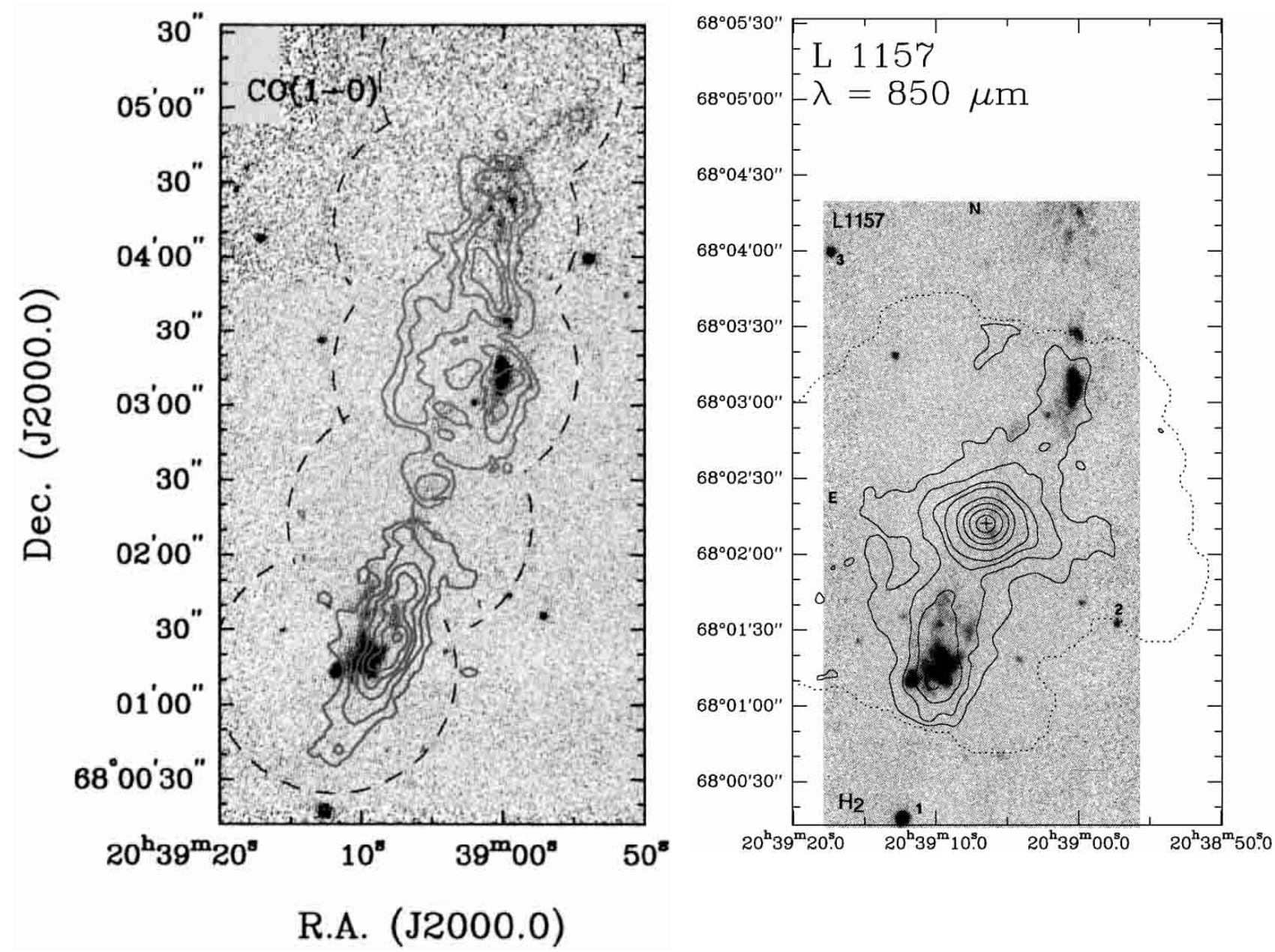

Fig. 3. Comparison between $\mathrm{CO}(1-0)$ and continuum emission. The left plot showing an $\mathrm{H}_{2} v=1-0 S(1)$ image overlaid with $\mathrm{CO}(1-0)$ contours is taken from Zhang et al. (2000). The image on the right displays an $\mathrm{H}_{2} v=1-0 S(1)$ image taken from Davis \& Eislöffel (1995) overlaid with contours of our $850 \mu \mathrm{m}$ measurement

isolated Class 0 source with a luminosity of $\sim 11 L_{\odot}$ (Umemoto et al. 1992) driving a molecular outflow with a rich chemistry (Gueth et al. 1996, 1997, 1998; Bachiller \& Pérez Gutiérrez 1997a). The source was detected at the VLA by Rodriguez \& Reipurth (1998). An infrared outflow was detected by Davis \& Eislöffel (1995), one knot coinciding with the HH 375 flow while Maradones et al. (1997), Choi et al. (1999) and Gregersen et al. (2000) find evidence for infall.

Figure 1 shows the 450, 850 and $1300 \mu \mathrm{m}$ images of the $\mathrm{L} 1157$ region. The maps show a central source with extended structure in an approximately east-west direction. The 850 and $1300 \mu \mathrm{m}$ data also show low level emission that extends to the north and south. This low-level emission is aligned with the CO outflow first observed by Umemoto et al. (1992) and later confirmed by Zhang et al. (1995). Furthermore, it is the second case in our sample, where a coincidence between a submillimetre peak and a Herbig-Haro object (HH 375) is apparent.

Obviously, all three images display a compact central source and a diffuse, east-west extension which might be due to a circumstellar disk or envelope perpendicular to the outflow direction. An additional continuum emission component is aligned with the $\mathrm{CO}$ outflow at 850 (see Fig. 3) and $1300 \mu \mathrm{m}$ but not at $450 \mu \mathrm{m}$. The peak flux densities and integrated flux densities in a $10^{\prime \prime}$ radius aperture are listed in Table 1 as well as the measurements carried out within an elliptical aperture of $55^{\prime \prime} \times 30^{\prime \prime}$. The smaller size of aperture was chosen as it should give only the characteristics of the central source, whereas the larger aperture also covers the disklike envelope and might even better enclose the emission detected by IRAS. Figure 2 shows the SED of this source for the smaller aperture with a grey-body fit to the $100-1300 \mu \mathrm{m}$ flux densities yielding $T_{\text {dust }}=29 \mathrm{~K}$ and $\beta=1.2$. Our values of $L_{\mathrm{FIR}} / L_{\mathrm{smm}}=63$ (central source) and 25 (source and envelope) are consistent with the Class 0 designation of this source (Bachiller \& Pérez Gutiérrez 1997a).

The reason for the discrepancy in the three maps concerning the non-detection of the southern outflow within the $450 \mu \mathrm{m}$ waveband, although it is clearly visible at $850 \mu \mathrm{m}$ and $1300 \mu \mathrm{m}$, might be found in the standard deviations listed in Table 2 . While the noise in the $850 \mu \mathrm{m}$ and $1300 \mu \mathrm{m}$ maps is extremely low, the value at $450 \mu \mathrm{m}$ 
is only average. Because of this difference, we analyzed the submillimetre emission in more detail. The IRAS fluxes were distributed by the already mentioned procedure of adopting the same flux ratio between the total source and the southern outflow as measured in the submillimetre regime. A grey-body fit then revealed that the $450 \mu \mathrm{m}$ flux lies just below the $3 \sigma$ detection limit. Therefore, the noise argument can easily solve the puzzle.

Another explanation for the relative radiative strength at 850 and $1300 \mu \mathrm{m}$ might have been an extreme contamination by strong molecular line emission. We therefore compared the $1300 \mu \mathrm{m}$ continuum flux with the CO (2-1) measurements carried out by Bachiller \& Pérez Gutiérrez (1997b). However, by adopting the equation

$\frac{F_{\text {line }}}{F_{\text {total }}} \approx 0.095 \frac{I_{\mathrm{CO}(2-1)}}{S_{1300}}$

introduced by Braine et al. (1995), we derived a line flux contamination originating from that transition of about $10 \%$, being typical for dusty environments. One would also expect lower rotational energy levels to be stronger populated than higher levels which also affects the transition probability. The contribution to the $450 \mu \mathrm{m}$ continuum flux by line emission (e.g. CO (6-5)) is therefore even more insignificant than for the $1300 \mu \mathrm{m}$ continuum flux. This may also be partly responsible for the non-detection of the bipolar outflow at $450 \mu \mathrm{m}$.

Still the similarity between the spatial CO distribution and our continuum maps remains obvious, so a closer relationship between the outflow of $\mathrm{CO}$ gas and the dust is very likely and interaction is almost certain. Umemoto et al. (1992) and Zhang et al. (2000) obtain a total gas mass of $\sim 0.1 M_{\odot}$, inferred from ${ }^{12} \mathrm{CO}(1-0)$ observations. If we adapt our continuum measurements $\left(S_{850}=1.1 \mathrm{Jy}\right.$, $\left.S_{1300}=0.2 \mathrm{Jy}\right)$ we derive a gas mass of $2 M_{\odot}$. This discrepancy could originate from the column density of $N\left(\mathrm{H}_{2}\right)=10^{21} \mathrm{~cm}^{-2}$ assumed by Umemoto et al. (1992). On the other hand, our calculations from the modified Planck fit yield $910^{21} \mathrm{~cm}^{-2}$. Further properties of the southern outflow derived from our calculations are a volume density of $410^{4} \mathrm{~cm}^{-3}$ and a dust temperature of $27 \mathrm{~K}$ which is warmer than the dust of central protostellar envelope. Although this value should be regarded with caution, it fits quite well into the interpretation of shock heating (Umemoto et al. 1992), which is barely detected in lowmass star formation regions. This scenario was introduced by Clark et al. (1986), who claimed that collisional heating of the dust by hot gas may be responsible for its FIR emission. Nevertheless, this source needs further investigation at mid- and far-infrared wavelengths with high spatial resolution in order to attain a proper temperature determination.

\subsection{IRAS $22134+5834$}

IRAS $22134+5834$ is located in Cepheus at an adopted distance of 900 pc (Dobashi et al. 1994a). It is a mediumto-high mass young star, with a luminosity of $\sim 1580 L_{\odot}$, embedded in a molecular cloud of mass $70 M_{\odot}$ (Dobashi et al. 1994b). These latter authors also detected a compact molecular outflow centred on this source.

Figure 1 shows the 450, 850 and $1300 \mu \mathrm{m}$ images of the IRAS $22134+5834$ region. The images display an elongated structure, with a semi-major axis of $0.22 \mathrm{pc}$ and a semi-minor axis of $0.15 \mathrm{pc}$, and an aspect ratio of $3: 2$. The minor axis of this cloud is closely aligned with the direction of the CO outflow observed by Dobashi (1994b).

The peak flux densities and integrated flux densities in a $50^{\prime \prime}$ radius circular aperture are listed in Table 1. This size of aperture was chosen as it enclosed all of the extended flux above the $3 \sigma$ level. This should be comparable to the IRAS PSC measurements. Figure 2 shows the SED of this source with a grey-body fit to the 100$1300 \mu \mathrm{m}$ flux densities yielding $T_{\text {dust }}=45 \mathrm{~K}$ and $\beta=1.0$. We note that our mass estimate is in good agreement with the $70 M_{\odot}$ found by Dobashi et al. (1994b). As far as we can tell from the available data, we estimate that it is most likely a Class I source.

\subsection{IRAS $23011+6126$}

IRAS $23011+6126$ is located in the Cepheus complex at an adopted distance of $730 \mathrm{pc}$, derived from a distance modulus of 9.3 quoted by Crawford \& Barnes (1970), in a small cloud known as Cepheus E. It contains a Class 0 source, with a molecular outflow discussed by Lefloch et al. (1996) and Ladd \& Hodapp (1997). Gregersen et al. (2000) report it as a likely candidate for circumstellar infall. Infrared observations of the $\mathrm{H}_{2}$ flow were made by Eislöffel et al. (1996). One of these knots is optically visible as HH 377. Ayala et al. (2000) conducted detailed spectroscopy on this HH object. The molecular outflow was also observed by Hatchell et al. (1999). Some limited millimetre observations were previously published by Bronfman et al. (1996) and Harju et al. (1993).

Figure 1 shows the 450, 850 and $1300 \mu \mathrm{m}$ images of the IRAS $23011+6126$ region. In the 850 and $1300 \mu \mathrm{m}$ images we see evidence of the quadruple outflow observed by Ladd \& Hodapp (1997). The elongation of the central source at the higher level contours in the north-south direction is coincident with an outflow detected in molecular hydrogen - as are the $450 \mu \mathrm{m}$ data. The southern lobe is coincident with $\mathrm{HH} 377$; so again at least part of the submm/mm emission might originate from dust embedded in the $\mathrm{HH}$ object. The low-level emission seen at 850 and $1300 \mu \mathrm{m}$ to the south-east (with a hint of very faint emission to the north-west) is coincident with an outflow detected in CO (see Ladd \& Hodapp 1997). The reason for its absence in the $450 \mu \mathrm{m}$ maps might again be a combination of a lack of sensitivity and rotational transition probability, as already shown for L 1157 in Sect. 3.8. Here again the noise at larger wavelengths is quite low (see Table 2), whereas its value at $450 \mu \mathrm{m}$ is extraordinarily high.

The peak flux densities and integrated flux densities in a $30^{\prime \prime}$ radius aperture are listed in Table 1 . This size 
of aperture was chosen as it encloses all of the extended flux from the central source and the north-south extended structure that is also seen in the NIR, and which we would also expect to have been detected by IRAS. However, this aperture size does not cover the area of the $\mathrm{CO}$ outflow. Therefore, the integrated flux measurement at 850 and $1300 \mu \mathrm{m}$ should be fairly reliable and comparable to the IRAS PSC measurements. Figure 2 shows the SED of this source with a grey-body fit to the 100$1300 \mu \mathrm{m}$ data yielding $T_{\text {dust }}=27 \mathrm{~K}$ and $\beta=1.5$. From the value $L_{\mathrm{FIR}} / L_{\mathrm{smm}}=67$ we corroborate earlier results that IRAS $23011+6126$ is a Class 0 source; however, the multiplicity of the source makes this estimate questionable.

\section{Conclusions}

We have presented new $\mathrm{mm} / \mathrm{submm}$ images of 10 regions associated with star formation as indicated by the presence of HH objects and embedded FIR sources. This has enabled us to classify a number of new and recently discovered young stellar objects. We have also made the first direct detections at $\mathrm{mm} / \mathrm{submm}$ wavelengths of $\mathrm{HH}$ objects themselves, and find enhanced dust emission from $\mathrm{H}_{2}$ and $\mathrm{CO}$ flows. Combining all of this information we reach the following conclusions:

1. For the first time, we detect Herbig-Haro objects at (sub)mm wavelengths. One of them ( $\mathrm{HH} \mathrm{8}$ ) possesses a gas column density of $4.410^{22} \mathrm{~cm}^{-2}$ and a dust temperature of $23 \mathrm{~K}$;

2. According to the ratio $L_{\mathrm{FIR}} / L_{\mathrm{smm}}$, we find that most compact sources investigated in the present paper appear to be in an extremely early stage of evolution;

3 . From the fact that several of the $\mathrm{mm} / \mathrm{submm}$ sources are associated with more than one VLA source we estimate that multiple star formation must be occurring in a significant number of sources;

4. The continuum emission that coincides with $\mathrm{CO}$ outflows may be triggered by shock heating caused by interaction of dust and emanating gas;

5. In the case of L 1527 and L 1157, where dust and CO contours are very similar, a contamination of the continuum flux by line emission originating from highly excited molecular transitions can be ruled out.

Due to the confusion in some of the regions and due to the presence of multiple VLA sources within the contours of a single $\mathrm{mm} / \mathrm{submm}$ source the need for high resolution data at FIR and $\mathrm{mm} / \mathrm{submm}$ wavelengths is obvious.

Acknowledgements. The authors wish to thank the staff of the JCMT, and in particular the telescope operators, for their support while these observations were carried out. We also gratefully acknowledge the British Council and the DAAD for financial travel support during the analysis of the data and the writing of this paper.

\section{References}

André, P., Ward-Thompson, D., \& Barsony, M. 1993, ApJ, 406, 122

Aspin, C., Sandell, G., \& Russell, A. P. G. 1994, A\&AS, 106, 165

Ayala, S., Noriega-Crespo, A., Garnavich, P. M., et al. 2000, AJ, 120, 909

Bachiller, R., Fuente, A., \& Tafalla, M. 1995, ApJ, 445, L51

Bachiller, R., \& Pérez Gutiérrez, M. 1997, ApJ, 487, L93

Bachiller, R., \& Pérez Gutiérrez, M. 1997, in Herbig-Haro Flows and the Birth of Low Mass Stars, ed. B. Reipurth \& C. Bertout, IAU Symp., 182, 152

Beichmann, C. A., Helou, G., \& Walker, D. W. 1988, Infrared astronomical satellite (IRAS), Catalogs and atlases, in (NASA Reference Press) ed. Beichmann et al.

Bontemps, S., André, P., Terebey, S., \& Cabrit, S. 1996, A\&A, 311,858

Braine, J., Krügel, E., Sievers, A., \& Wielebinski, R. 1995, A\&A, 295, L55

Bronfman, L., Nyman, L.-Å, \& May, J. 1996, A\&AS, 115, 81

Chandler, C. J., \& Richer, J. S. 2000, ApJ, 530, 851

Chen, H., Tafalla, M., Greene, T. P., Myers, P. C., \& Wilner, D. J. 1997, ApJ, 475, 163

Chini, R., Krügel, E., Haslam, C .G. T., et al. 1993, A\&A, 272, L5

Chini, R., Reipurth, B., Sievers, A., et al. 1997, A\&A, 325, 542

Choi, M., \& Lee, Y. 1998, ApJ, 498, L71

Choi, M., Panis, J. F., \& Evans II, N. J. 1999, ApJS, 122, 519

Chrysostomou, A., Hobson, J., Davis, C. J., Smith, M. D., \& Berndsen, A. 2000, MNRAS, 314, 229

Clark, F. O., Laureijs, R. J., Chlewicki, G., et al. 1986, A\&A, 168, L1

Codella, C., Bachiller, R., \& Reipurth, B. 1999, A\&A, 343, 585

Cohen, M., \& Schwartz, R. D. 1979, ApJ, 233, L77

Crawford, D. L., \& Barnes, J. V. 1970, AJ, 75, 952

Davis, C. J., \& Eislöffel, J. 1995, A\&A, 300, 851

Dent, W. R. F., Matthews, H. E., \& Ward-Thompson, D. 1998, MNRAS, 301, 1049

di Francesco, J., Evans II, N. J., Harvey, P., Mundy, L. G., \& Butner, H. M. 1998, ApJ, 509, 324

Dobashi, K., Bernard, J. P., Yonekura, Y., \& Fukui, Y. 1994, ApJS, 95, 419

Dobashi, K., Nozawa, S., Hayashi, Y., Sato, F., \& Fukui, Y. 1994, AJ, 107, 2148

Eiroa, C., Miranda, L. F., Anglada, G., Estalella, R., \& Torrelles, J. M. 1994, A\&A, 283, 973

Eislöffel, J., Mundt, R., \& Böhm, K.-H. 1994, AJ, 108, 1042

Eislöffel, J., Smith, M. D., Davis, C. J., \& Ray, T. P. 1996, AJ, 112,2086

Gomez, M., Whitney, B. A., \& Kenyon, S. J. 1997, AJ, 114, 1138

Greene, T. P., \& Lada, C. J. 1996, ApJ, 461, 345

Gregersen, E. M., Evans II, N. J., Zhou, S., \& Choi, M. 1997, ApJ, 484, 283

Gregersen, E. M., Evans II, N. J., Maradones, D., \& Myers, P. C. $2000, \mathrm{ApJ}, 533,440$

Griffin, M. J., Ade, P. A. R., Orton, G. S., et al. 1986, Icarus, 65,244

Griffin, M. J., \& Orton, G. S. 1993, Icarus, 105, 537

Grossman, E. N., Masson, C. R., Sargent, A. I., et al. 1987, ApJ, 320, 356

Gueth, F., Guilloteau, S., \& Bachiller, R. 1996, A\&A, 307, 891 
Gueth, F., Guilloteau, S., Dutrey, A., \& Bachiller, R. 1997, A\&A, 323, 943

Gueth, F., Guilloteau, S., \& Bachiller, R. 1998, A\&A, 333, 287

Hatchell, J., Fuller, G. A., \& Ladd, E. F. 1999, A\&A, 346, 278

Harju, J., Walmsley, C. M., \& Wouterloot, J. G. A. 1993, A\&AS, 98, 51

Herbig, G. H., \& Jones, B. F. 1983, AJ, 88, 1040

Hogerheijde, M. R., van Dishoeck, E. F., Blake, G. A., \& van Langvelde, H. J. 1997, ApJ, 489, 293

Hogerheijde, M. R., van Dishoeck, E. F., Blake, G. A., \& van Langvelde, H. J. 1998, ApJ, 502, 315

Holland, W. S., Cunningham, C. R., Gear, W. K., et al. 1998, in Advanced Technology MMW, Radio, and Terahertz Telescopes, ed. T. G. Phillips, SPIE, 3357, 305

Kenyon, S. J., Dobrzycka, D., \& Hartmann, L. 1994, AJ, 108, 1872

Krusch, E. 1999, ISOPHOT Beobachtungen protostellarer Kondensationen, Diplomathesis, Astronomisches Institut der Ruhr-Universität Bochum, airub99046

Ladd, E. F., \& Hodapp, K.-W. 1997, ApJ, 474, 749

Lefloch, B., Eislöffel, J., \& Lazareff, B. 1996, A\&A, 313, L17

Maradones, D., Myers, P. C., Tafalla, M., et al. 1997, ApJ, 489, 719

Molinari, S., Brand, J., Cesaroni, R., \& Palla, F. 1996, A\&A, 308,573

Molinari, S., Ceccarelli, C., White, G. J., et al. 1999, ApJ, 521, L71

Mouri, H., \& Taniguchi, Y. 2000, ApJ, 534, L63

Myers, P. C., Fuller, G. A., Mathieu, R. D., et al. 1987, ApJ, 319,340

Ohashi, N., Hayashi, M., Kawabe, R., \& Ishiguro, M. 1996, ApJ, 466, 317

Ohashi, N., Hayashi, M., Ho, P. T. P., \& Momose, M. 1997, ApJ, 475, 211

Onishi, T., Mizuno, A., Kawamura, A., Ogawa, H., \& Fukui, Y. 1998, ApJ, 502, 296

Orton, G. S., Griffin, M. J., Ade, P. A. R., et al. 1986, Icarus, 67,289
Pravdo, S. H., Rodríguez, L. F., Curiel, S., et al. 1985, ApJ, 293, L35

Reipurth, B. 1989, Nature, 340, 42

Reipurth, B., \& Eiroa, C. 1992, A\&A, 256, L1

Reipurth, B., Chini, R., Krügel, E., Kreysa, E., \& Sievers, A. 1993, A\&A, 273, 221

Reipurth, B., Nyman, L.-A., \& Chini, R. 1996, A\&A, 314, 258

Reipurth, B., Bally, J., \& Devine, D. 1997, AJ, 114, 2708

Reipurth, B., Yu, K. C., Rodriguez, L. F., Heathcote, S., \& Bally, J. 1999, A\&A, 352, L83

Reipurth, B., Yu, K. C., Heathcote, S., Bally, J., \& Rodríguez, L. F. 2000, AJ, 120, 1449

Rodríguez, L. F., Escalante, V., Lizano, S., Canto, J., \& Mirabel, I. F. 1990, ApJ, 365, 261

Rodríguez, L. F., Anglada, G., \& Curiel, S. 1997, ApJ, 480, L125

Rodríguez, L. F., \& Reipurth, B. 1998, Rev. Mex., 34, 13

Rodríguez, L. F., Anglada, G., \& Curiel, S. 1999, ApJS, 125, 427

Sandell, G. 1994, MNRAS, 271, 75

Strom, S. E., Strom, K. M., Grasdalen, G. L., et al. 1985, AJ, 90, 2281

Tamura, M., Gatley, I., Waller, W., \& Werner, M. W. 1991, ApJ, 374, L25

Tamura, M., Ohashi, N., Hirano, N., Itoh, Y., \& MoriartySchieven, G. H. 1996, AJ, 112, 2076

Umemoto, T., Iwata, T., Fukui, Y., et al. 1992, ApJ, 392, L83

Viotti, N. R. 1969, Mem. Soc. Astron. Ital., 40, 75

Ward-Thompson, D., Chini, R., Krügel, E., Andre, P., \& Bontemps, S. 1995, MNRAS, 274, 1219

Wilking, B. A., Blackwell, J. H., Mundy, L. G., \& Howe, J. E. 1989, ApJ, 345, 257

Zhang, Q., Ho, P. T. P., Wright, M. C. H., \& Wilner, D. J. 1995, ApJ, 451, L71

Zhang, Q., Ho, P. T. P., \& Wright, M. C. H. 2000, AJ, 119, 1345

Zhou, S., Evans II, N. J., \& Wang, Y. 1996, ApJ, 466, 296 